\begin{tabular}{ccc}
\hline & International Journal of Current Research in \\
Biosciences and Plant Biology & Volume $5 \bullet$ Number 10 (October-2018) ・ ISSN: $2349-8080$ (Online) \\
\hline EXCELLENT \\
PUBLISHERS
\end{tabular}

Original Research Article

doi: https://doi.org/10.20546/ijcrbp.2018.510.006

\title{
Genus Curcuma L. (Zingiberaceae) from Maharashtra State - India
}

\author{
A. S. Jadhao* and A. S. Bhuktar
}

Vivekanand Arts, Sardar Dalipsingh Commerce and Science College, Samarth Nagar, Aurangabad, Maharashtra - 431 001, India

*Corresponding author.

\begin{tabular}{l}
\hline Article Info \\
\hline Date of Acceptance: \\
24 September 2018 \\
Date of Publication: \\
06 October 2018 \\
\hline Key w ords \\
\hline Curcuma \\
Maharashtra \\
Spice family \\
Zingiberaceae
\end{tabular}

ABSTRACT

Genus Curcuma L. belongs to family Zingiberaceae. A floristic study of genus Curcuma L. from the Maharashtra state - India, has been carried out from June 2013 to August 2016. The samples of the Curcuma species were collected from the different localities of the state during the monsoon season. The samples were proposed to herbarium and identified on the basis of morphological features. 11 species of the genus were identified viz., Curcuma amada Roxb., C. angustifolia Roxb., C. aromatica Salisb., C. caulina J. Grahm., C. decipiens Dalz., C. inodora Blatt., C. longa L., C. neilgherrensis Wight, C. pseudomontana J. Grahm., C. scaposa (Nimmo) Skornick. \& M. Sabu and C. zedoaria (Christm) Roscoe.

\section{Introduction}

Zingiberaceae Adans. is established with type genus Zingiber Boehm., and popularly known as spice family because it includes many spices like turmeric, ginger and cardamom etc. known for its immense values as food, medicinal, ornamental, traditional and cultural. The valid number of genera is 53 with about 1200 species (Kress et al., 2002). Zingiberaceae is a largest of 8 families in order Zingiberales (Naïve, 2017), India represents 21 genera and about 200 species whereas in Maharashtra state 11 genera and about 29 species (Sharma et al., 1996). In India Zingiberaceae is mostly distributed in South India, Northeast India and Andaman \& Nicobar Island.

Curcuma L. is one of the largest genera in Zingiberaceae which comprises of approximately 120 species and distributed mainly in the tropics of Asia from India to South China, Southeast Asia, Papua New Guinea and Northern Australia. Tropical Asia and South Asia are the diversity hotspots of the genus (Chen et al., 2015). Skornickova and Sabu (2002) revised the genus and recorded 29 species from India. But recently India accounts 35 species. Sabu (2006) has done floristic studies on the Zingiberaceae and Costaceae of 
South India and recorded 20 species of Curcuma L. from South India. Sharma et al. (1996) recorded 10 species from Maharashtra state. Curcuma L. is an economically important genus; it has been used frequently as spices, dyes and as medicine (Chen and Xia, 2013). However the genus is very difficult for its taxonomic identity because of its extremely short period of flowering, and also difficult for the herbarium preparation due to its flashiness of tubers, rhizomes and inflorescence.

\section{Materials and methods}

The sample of Curcuma L. species were collected from the different localities of Maharashtra state, the exiccata of plant species are given along with date of collection and field number. The detail morphological characters were studied by using floristic literature (Cooke, 1958; Naik, 1998; Sharma et al., 1996; Sabu, 2006; Yadav and Sardesai, 2002). The herbariums of each species were prepared and voucher specimens are deposited in VH Herbarium, Department of Botany, Vivekanand Arts, Sardar Dalipsingh Commerce and Science College, Samarth Nagar, Aurangabad.

\section{Results}

\section{Curcuma amada Roxb.}

Vernacular names: Marathi: Aambe halad, Hindi: Amba haldi, Kannada: Amba-halad, Amba haldi, Malayalam: Mannayinci, Tamil: Arukamleke, Mankayinci, Telgu: Mamidiallan.

Herbs, leafy shoot 2-3 feet or more. Rhizome large, yellow inside having smell of green mango 3$5.5 \times 2-3.2 \mathrm{~cm}$., sessile tuber cylindrical, branched, horizontal $5-8.5 \times 2-3 \mathrm{~cm}$. Root tuber is absent. Leaves 4-6, large, hairy 0r glabrous, oblong, lanciolate, acute, narrowed at base $40-65 \times 12-15 \mathrm{~cm}$; petiole as long as leaf lamina. Inflorescence central; peduncle $10-15 \mathrm{~cm}$ or more; spike $7.5-12.5 \mathrm{~cm}$ long; coma bracts fused at base, light green 6-8; fertile bracts 15-20, orbicular, obtuse, and slightly recurved. Flower as long as bracts; calyx 3 lobed at tip, cleft one side, $1 \mathrm{~cm}$ long; corolla whitish, pale yellow, corolla tube funnel shaped, $2.5-3 \mathrm{~cm}$ long; labellum 3 lobed, pale yellow with dark yellow bands; lateral staminode pale yellow, slightly recurved at apex; stigma appressed within anther lobes; style long, filiform. Ovary trigonous, tricarpellary, syncarpous, ovules many, 3mm long, fruiting not seen (Fig. 1a).

* Type: Iconotype: Roxburgh, Ic. T. 1760 (CAL).

Distirb.: Buldhana, Konkan (Cooke, op.cit.)

Fls. \& Frts: June - September.

Exsiccata: Khamgoan Buldhana ASJ 7620

Specimen examined: B H Mumbai, Victoria garden, July 1917, 32710; BAMU: Nanded, Amboli 07.09.1979, B. R. Zate, Zate 920; Khari, 05.09.1979, B. R. Zate, Zate B. R. 741.

\section{Curcuma angustifolia Roxb.}

Vernacular names: English: Narrow-leaved turmeric; Manipuri: Yaipan, Hindi: Tikhur, Malayalam: Koova.

Herbs, root fiber numerous, slender; root tubers small, ellipsoid, pale yellow, white inside, 1-3 $\times 0.5-1-3 \mathrm{~cm}$. Rhizomes ovoid or conical, 1.2-5.5 $\times 0.5-1.7 \mathrm{~cm}$, white inside, having pungent smell. Leafy shoot 50 $120 \mathrm{~cm}$ tall; leaves 3-4, elliptic, oblong, acuminate, narrowed at both ends, $30-65 \times 9-15 \mathrm{~cm}$; petiole with sheath, 10-45 cm long. Flower in terminal or lateral spikes 9-15 × 3-5 cm long; 3-3.5 cm, yellow, 1-2 in each bracts; peduncle 15-30 cm long; floral bracts ovate- oblong, 3-4 cm long, obtuse or rounded at tips with dark purple spot; coma bracts oblong, purple, base white, minutely pubescent, $4-5.5 \mathrm{~cm}$ long; calyx white, 3 lobed, hairy outside, $1 \mathrm{~cm}$ long, lobes oblong, obtuse; corolla tubes funnel shape, white, $2.5-3 \mathrm{~cm}$, lip yellow obovate, acuminate as long as corolla; lateral staminodes obovate - cuneate; stamens 5-7 $\mathrm{mm}$, anther thecae parallel 4-5 $\mathrm{mm}$ long, spurred. Ovary trilocular, hairy, ovules 2 in each locules; capsule ovoid, 7-10 mm long, seed globose, reddish brown (Fig. 1b). 
* Type: Lectotype: Asiat. Res. (Calcutta) 11: Tab. 3. 1810 .

Distirb. : Nanded, Pune

Fls. \& Frts.: June-September.

Exsiccata: Shitakhandi, Nanded, ASJ 7615.

Specimen examined: B H Mumbai: 27 June 86, D. K. Patel, 74126. BAMU: Nanded, Ambadi, 09.07.1971, Naik V. N., Naik 1151; Kinwat, 08.07.1971, Naik V. N., Naik 1133; Sitakhandi, 03.07.1979, M. A. W. Khan, Khan 33.

\section{Curcuma aromatica Salisb.}

Vernacular names: English: Wild turmeric, aromatic turmeric, Marathi: Ran halad, Hindi: Jangali halad, Manipuri: Lam yaingang, Tamil: Kasturimanjal, Telugu: Kasthuri pasupa, Kannada: Kasthuri arishina.

Herbs, rhizome small, $1.2-2.5 \times 0.5-1 \mathrm{~cm}$, pale yellow within, strongly aromatic, sweet camphoraceous smell. Root fiber many, sessile tubers many, ellipsoid, almond like 1.5-2.5 × 0.6$1 \mathrm{~cm}$. leafy shoot $22-35 \mathrm{~cm}$ long; leaves distichous 4-6; lamina 9.5-18.5 × 4.5-7 cm, lanciolate, oblong- elliptic, acuminate and densely pubescent bellow; petiole as long as or longer than lamina. Inflorescence central $10-15 \times 2-3.5 \mathrm{~cm}$; peduncle covered by sheath $5-6.5 \mathrm{~cm}$ long; coma bracts large, pink; fertile bracts $5.5 \mathrm{~cm}$ long, pale greenish, tips recurved, slightly hairy; bracteoles white, sparkly pubescent, up to $2 \mathrm{~cm}$ long; calyx sparkly pubescent, tip 3 lobed $2 \mathrm{~cm}$ long; corolla pinkish white, funnel shaped, lobes unequal; labellum deep yellow, orbicular, 3 lobed; lateral staminodes as long as corolla lobes, oblong, obtuse; anther thecae parallel, long, sharp spur at base; style filiform, long; stigma bi-lobed having perforation in center; capsule globose, 3-valved; seed ovoid, light brown, $2 \mathrm{~mm}$ long, aril white (Fig. 1c).

*Type: Iconotype: Roxburgh, Ic. t. 1010 (CAL).
Distrib: Bombay, Satara, Sindhudurg.

Fls. \& Frts.: May - July

Exsiccata: Sawantwadi Narendra Pahad, Sindhudurg. ASJ 7618

Specimen examined: B H Mumbai: Thane, Bassein, 28.10.1907, HPP. 176/9/3; Dapoli, 1922, R. D. Garad, ACK 1184; Yellapus Sahashrahalli Khar, N. K. 26.05.1954, H. Santapau S.J., 18723; Mumbai, Andheri National caves, 8 Aug. 1942, HS.668; Borivali, Kavery Caves, 25.08.45, H. S., HS. 7059 BSI: Rajastan Bijalia hills, Khushlgarh Banswara, 12.10.61, K. Chandra, 75700.

\section{Curcuma caulina J. Grahm.}

Vernacular names: English: Indian arrowroot, Arrowroot lily, Marathi: Chavar, Kachar, Sitachavari, Hindi: Tikhur.

Herbs, root fibers numerous, slender, long; root tubers small, oval, elliptic, pale brown and white inside $2-6 \times 0.8-1.6 \mathrm{~cm}$. Rhizome small, ovoid or conical, pale yellow or pale brown inside, 3-5.5 $\times$ $1-1.5 \mathrm{~cm}$. leafy shoot $1-1.5 \mathrm{~m}$ tall; leaves $5-6,25-55$ $\times 5-12.5 \mathrm{~cm}$ long, oblong, oblong lanciolate, acuminate, narrowed at base; petioles sheathing. Inflorescence central or terminal; peduncle 20-30 cm long; spike $10-18 \times 3.5-5 \mathrm{~cm}$ long; flower pale yellow or white $4-5 \mathrm{~cm}$, longer than bracts; coma bracts pale yellow or white oblong, lanciolate; fertile bracts oblong lanciolate, pale green, violet in colour, 4-5 × 2-2.5 cm; calyx pale yellow or white $1-1.5 \mathrm{~cm}, 3$ lobed at tip; corolla tube funnel shaped $4.5-5 \mathrm{~cm}$ long, white; labellum white 3 lobed, deflected; stamen 5-6 mm, anther thecae parallel 4$5 \mathrm{~mm}$ long; capsule oblong, coriaceous; seeds ovoid with lacerate aril (Fig. 1d).

*Type: Neotype: India, Maharashtra, Mahabaleshwar, below Nakinda Village.

Distrib: Pune, Satara

Fls. \&Frts.: July- November 
Exsiccata: Pachgani-Mahabaleshwar Satara, ASJ 7617

\section{Specimens examined:}

BH Mumbai: $H$. caulina Maharashtra, Lingmala, 20.06.1958, P. V. Bih, P.V.1663; Satara, Mahabaleshwar, 07.11.50, Santapau, Santapau 11831; 11879; Mahabaleshwar, 17.10.54, P.V. Bole, Bole 1202; Mahabaleshwar, Oct. 1924, A.C.K., ACK 1183; Mahabaleshwar, 15.09.54, B. Rukmini, 236 B.R., 233 B.R.; Faisalabad ghat, 31.08.59, BB364; Kas Plateau, 1 Aug. 1968, P.V. Bole, Bole 1690; BSI Pune: Pune, Mahabaleshwar, lake point, 12.10.60, M. Y. Ansari, U. R. Deshpande, 67681. S U K: Kolhapur District, Kas, Aug. 91, M. P. Bachulkar 5132; M. M. Sardesai mms-1495; Satara, Mahabaleshwar, Gaikwad S. P. 524; Mahabaleshwar, Priti D. Mahekar, SVK.

\section{Curcuma decipiens Dalz.}

Vernacular Name: Marathi: Jambhali ranhalad, Hindi: Jangli haldi, van haldi, Malayalam: Kuzhi kua.

Herbs, root stock short, tuber numerous, almond like; ellipsoid, white inside, $2.5-3.8 \times 1-1.8 \mathrm{~cm}$. Rhizome small, ovoid, conical, pale yellow- white within, 3$6 \times 1-1.3 \mathrm{~cm}$.Leafy shoot $35-45 \mathrm{~cm}$ tall; leaves $4-6$, distichous, petiolate, $17-25 \times 5.5-9.5 \mathrm{~cm}$, broadly elliptic, rounded at base, membranous, deltoid, acuminate; petiole as long as lamina. Inflorescence central; peduncle $15-20 \mathrm{~cm}$; spike 8-12 × 2-3.5 cm; coma bracts many, green- purple; flower twin, 2.5-3.5 $\mathrm{cm}$ long, flowering bract $2.5-3 \mathrm{~cm}$ long, saccate greenish purple; coma bracts numerous, purple, oblong, obtuse or rounded at the apex, 3.5-4 cm long; calyx 3 lobed, puberlose outside $10-12.5 \mathrm{~mm}$; corolla tube $2-2.5 \mathrm{~cm}$, funnel shaped, lobe oblong, lanciolate, deep purple with white base, margin crisped; anther thecae parallel, spurred having purple spot; stigma bilipped, style long, ovary trilocular, seeds many, globose, brown, 2.5-3mm (Fig. 1e).

*Type: Lectotype: [India, Maharashtra] Bombay, Dalzell s.n. (K!).
Distrib.: Amravati, Bombay, Kolhapur, Raigad, Ratnagiri, Sindhudurg, Thane

Fls. \& Frts.: June - September

Exsiccata: Lanja Ratnagiri. ASJ 7621

\section{Specimen examined:}

B. H. Mumbai: Mumbai, Vajreshwari, 04.08.55, B. Rukminibai, B.R.341, 441, 342, 439, 440, R 1977; Mulgoan, Salsette Isl, 15 June 1941, H. Santapau S.J., 22Q.1H, Marrehikeri N. Kanara, 28.05.50, JF.1575. BAMU: Nanded, Dudhad, Bhokar, 31.08.1981, M. A. W. Khan, 1780; Sitakhandi, 03.08.1980, M. A. W. Khan, 918; Amboli, 19.08.1981, B. R. Zate, Zate 1962; Kharbi, 16.08.1981, B. R. Zate, Zate 1846. BSI Pune: Amravati, Raipur, 25.08.76, M. Y. Ansari, 143936; Chandrapur, Allapalli, Talwada village, 11.09.1963, R. S. Rao, 91543; Vaitarna forest, 24.08.67, K. V. Billore, 110473-A; Pune, Bhimashankar, 20.06.56, G. S. Puri \& Moorthy, 2244; Devchirai-Phonda, 03.10.70, B. G. Kulkarni, 121373. SUK: Kolhapur District, M. M. Sardesai, mms -1410.

\section{Curcuma inodora Blatt.}

Vernacular name: 'Scentless turmeric', 'hidden lily'.

Herbs; root tuber numerous oblong, ellipsoid, white inside $2-3.5 \times 1-1.5 \mathrm{~cm}$. Rhizome small, conical, pale yellow inside having no odour, 2-3 $\times 2-2.5 \mathrm{~cm}$. Leafy shoot $20-30 \mathrm{~cm}$ tall; leaves radical, 5-7, petiolate, broadly elliptic, lanciolate, tip acuminate, plicate; lamina $10-15 \times 5-7.5 \mathrm{~cm}$ long, winged, deeply concave. Inflorescence central; peduncle 5-7 cm long; Spike 5-10 × 2.5-3cm long; flower 2-3 $\times$ $1-1.5 \mathrm{~cm}$; coma bracts violet, tip rounded, 3-3.5 $\times 1$ $1.5 \mathrm{~cm}$ long; calyx 3 lobed at apex, 1-1.2 cm white; corolla purplish with a yellow streak on the lip; corolla tube $1.5-2 \mathrm{~cm}$; anther thecae parallel, white having pink base, spur bent inwards; stigma bilipped; style long, filiform; ovary trilocular with many ovules; seeds many, ovoid, arillate, brown, 2.5-3mm (Fig. 1f). 


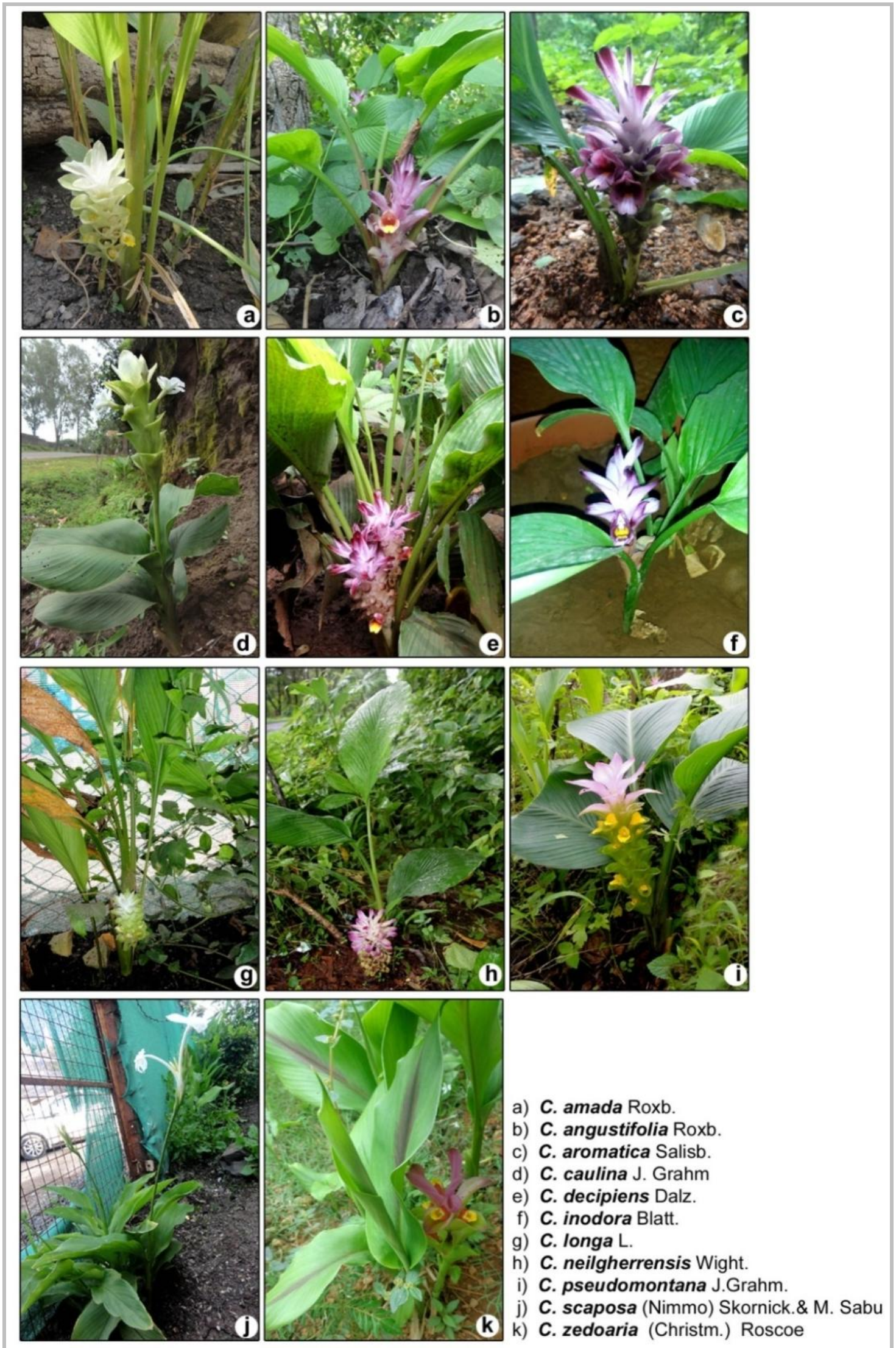

Fig. 1: The genus, Curcuma L. in Maharashtra State, India. 
*Type: Bombay Prasidency, Moogaum, Salsette, Halberg 12724 (Blat.)

Distrib.: Bombay, Buldhana, Kolhapur, Nashik, Raigad, Ratnagiri, Thane.

Fls. \& Frts.: June - November.

Exsiccata: Ajara town Kolhapur. ASJ 7622.

Specimen examined: B H Mumbai: Maharashtra Mumbai, 22.7.1954, K.V.S., K.V.S. 390; Mumbra, 07.07.1953, K. V. Sheuory, K.V.S. 19; Malad Podar Chaels, 07.08.54, Shah, Shah 150, 156; Girvas hills, Junagadh, 25.08.52, Bole, Bole 696; Mulgoan, Salsette Isl, 15 June 1941, 229.2H; Khandala St. X. Villa, 30.06.54, B. Rukminibai, 157 B.R.; Matheran, 08.08.54., B. Rukmini, 190 B.R. BSI Pune: Bhandara, Hazara falls, nursery side, 01.10.76, S. M. Malhotra, 148980; Buldhana, Hajam nala, Ambaborvani, 20.6.82, P. G. Diwakar, 162811; Thane, Bhargad, Utwada hills, 20.10.76, K. V. Billore, 112979; Kedarnath hill slope, 06.11.68, K. V. Billore, 115806; Denga Chi Met. R.F., 20.07.68, K. V. Billore,111734; Ghotarna Mohili R.F., 20.07.68, K. V. Billore, 116258; Kedarnath hills, 20.11.68, K. V. Billore, 115806. S U K: Kolhapur District, M. M. Sardesai, MMS 172.

\section{Curcuma longa L.}

Vernacular name: English: Turmeric, Hindi: Haldi, Marathi: Halad, Sanskrit: Haridra, Tamil: Manjal, Telagu: Pasapu, Kannada: Arishina.

Herbs, sessile root tubers many, thick, cylindric, branched, orange- yellow inside, aromatic; root tubers rare. Rhizomes conical, deep orange-yellow inside, 3-6 $\times 2-3 \mathrm{~cm}$. Leafy shoot 4-5.5 feet tall; leaves 6-8, distichous, oblong, lanciolate, tapering to the base, entire, acute or acuminate, 45-100 $\mathrm{x}$ $13-20 \mathrm{~cm}$ or more; petiole with sheath and shorter than the leaf lamina, 20-35 cm long, ligules short near the lamina. Inflorescence central 12-15 $\times 3$ $5 \mathrm{~cm}$; peduncle $7-15 \mathrm{~cm}$ concealed within the sheath; spike $10-15 \mathrm{~cm}$ long, with distinct white coma; flowering bracts pale green, ovate, $2.5-4 \mathrm{~cm}$ long; coma bracts 10-14, 5.5-6cm long, oblong, obtuse; flower $4.5 \mathrm{~cm}$ long, equal to bracts; calyx scarcely 3-lobed, 2-2.5 cm long, white; corolla shortly longer with oblong lobes; lip 3-4cm long, bright yellow; anther thecae spurred, 3.5-4 mm long; ovary hairy, tricarpellary, ovules many, fruiting not seen (Fig. 1g).

*Type: Lectotype: Manjellakua Rheede, Hort. Malab. 11:21. T. 11. 1692.

Distrib.: Cultivated for the turmeric obtained from rhizomes but is doubtfully wild (Cook, op.cit.). Aurangabad, Buldhana, Kolhapur, Nagpur, Osmanabad, Satara, Sangali, Sindhudurg.

Fls. \& Frts.: June - November.

Exsiccata: Gangapur Aurangabad. ASJ 7616.

Specimen examined: B H Mumbai: Maharashtra, Mumbi, Bassein, 20 Aug. 1902, G. A. Rayn, GA 266, 259; Poona, Agriculture college Poona, 11. 06. 1955, B. Rukminibai, B.R. 471, 467, 469.

\section{Curcuma neilgherrensis Wight.}

Vernacular name: English: Narrow leaved turmeric, East Indian Arrow root, Bengali: Keturi haludhi Hindi: Tikhur, Marathi: Tavakeera, Malayalam: Kattumanjal, Koova, Vellakkua, Manipuri: Yaipan.

Herb, root fiber numerous, slender, root tuber small and white inside. Leafy shoot $25-35 \mathrm{~cm}$ tall; leaves 4-6, shortly petiolate, lanciolate or oblong lanciolate, acuminate narrowed at base, 15-25 $\times$ $8.5-9.5 \mathrm{~cm}$. Inflorescence lateral, appears before the leaves $10-15 \times 4-5 \mathrm{~cm}$; coma bracts 5-7 oblong lanciolate, pink or purple; fertile bracts oblong lanciolate, pale yellowish green; flower is $4-4.5 \mathrm{~cm}$ bright yellow; calyx 3 lobed violet dotted 0.8$1.2 \mathrm{~cm}$ long; corolla tube $1.5 \mathrm{~cm}$ long, lobes oblong, whitish yellow; anther thecae parallel, spurred, 
3.5-4mm long; style long, filiform, bilipped; ovary trilocular, ovules many; capsule c $1.3 \mathrm{~cm}$ in diameter; seeds ovoid or globose, arillate, brown 3-4mm long (Fig. 1h).

*Type: Lectotype: Wight s.n. (K!);

Distrib.: Amaravati, Kolhapur, Pune, Sindhudurg.

Fls. \& Frts.: September - November.

\section{Exsiccata: Radhanagari Kolhapur. ASJ 7623}

Specimen examined: B H Mumbai: Maharashtra, Mumbai, Malgoan Salsette, 3.07.41, H.S., HS.229.3H, 229. 4H. BSI Pune: Amaravati, Way to Bairat, 22.06.77, M. Y. Ansari, 146818; Belghat, 28.06.77, M. Y. Ansari, 149318; Pune, Lonavala, 14.05.64, Hemadri K, Jadhav C. R. 89936; Chipalun forest, Koyna nagar Rd, 28.12.61, V.N. Naik, 4958, Ratnagi, Shivapur, 05.06.70, B. G. Kulkarni, 121045; Bedshi, 27.05.70, B. G. Kulkarni, 120902; Semadoh, 24.06.77, M. Y. Ansari, 141721. SUK: Kolhapur District, Kas, June 91, M. P. Bachulkar 510;, M. M. Sardesai, 51; Ramghat, 19.10.91, A. S. Narvekar, 150; Patgoan, 3.08.1998, S. R. Yadav, 5814.

\section{Curcuma pseudomontana J. Grahm.}

Vernacular name: English: Hill turmeric, Hindi: Kachura, Marathi: Raan halad, Shindalavana, Tamil: Kattu manjal, Malayalam: Kattu manjal.

Herbs, root stock small having ovoid or subglobose tubers, yellow in center and white periphery, $2.5-5 \times 1.5-2 \mathrm{~cm}$. Rhizomes small, conical or cylindrical, 3-6 × 1-2 cm, pleasantly aromatic, yellowish at center and white periphery. Leafy shoot $70-110 \mathrm{~cm}$ tall; leaves $4-6$, distichous, oblong lanciolate, acuminate, tapering to base; petiole longer than lamina; lamina $22-40 \times 7.5-14$ $\mathrm{cm}$. Inflorescence both lateral and central up to $15-20 \times 3.5-4.5 \mathrm{~cm}$; spike $8-10 \mathrm{~cm}$ with distinct coma; coma bracts 4-5, elliptic, tip rounded, bright pink, purple, white or other shades of color; fertile bracts 10-16, bracteole pink, 4-6 mm long; flower longer than bracts, bright yellow; calyx pale yellow $2-2.5 \mathrm{~cm}$ long, 3-lobed at tip; corolla tube funnel shaped, $3.5-4 \mathrm{~cm}$ long with oblong acute lobes; labellum 3 lobed, bright yellow, deflexed; anther thecae parallel, $4 \mathrm{~mm}$ long, spurred, pointed forward; stigma slightly exerted to the anther lobes; style long, filiform; ovary tricarpellary, cylindrical, ellipsoid, 4-5mm long; ovules many; capsule ellipsoid- ovoid; seeds many, globose, pale brown, arillate 1-2 × 1$1.5 \mathrm{~mm}$ (Fig. 1i).

*Type: Neotype: India, Maharashtra, Pune District, Khandala, slopes around St. Xavier's villa, 19.VI.2003, Sornickova 73402.

Distrib.: Ahmednagar, Akola, Amaravati, Aurangabad, Bhandara, Bombay, Chandrapur, Kolhapur, Nagpur, Nasik, Pune, Raigad, Satara, Thane.

Fls. \& Frts.: June - September.

Exsiccata: Mhaismal Aurangabad. ASJ 7619.

Specimen examined: B $\mathbf{H}$ Mumbai: Maharashtra, Poona, Ganeshkhind garden, 29.09.1910, R. G. Javlekar, 74170, 74164; Mumbai, National park, Borivali, 3 July 1955, B. Rukminibai, R1942; Aavery Milk Colony, Goregoan, 22.06.1958, S. T. Tavakar, T-1240; Khandala, St. X. Villa, 18.07.1957, H. Santapau S.J., 21889; Matheran, 3 Oct. 1960, N. A. Irani, NI 5465, 4152; BAMU: Aurangabad, Mhaismal, Pokle, Pokle 4546; Lonavala, 15.09.1967, H. Kamlakar, BSI Pune: Bhandara, Chorkmal forest, 21.09.76, S. K. Malhotra, 144672, Chandrapur, Jamni Rd, 21.11.73, B.M. Wadhwa, 137246; Khandala, 15.06.56, S. K. Jain, 2280; Nasik, Harkol, 20.08.196, B. G. Kulkarni, 105631; Pune, Bhimashankar Rd., 08.10.62, K. P. Janardhanan, 81696; Lonavala, 27.06.64, R. Venkata Reddi, 97956; Katraj, 16.6.1956, G.S. Puri, R. M. Patil, 2358; Matheran view point, 28.07.66, B. H. Wadhwa, S. K. Mudliar, 109826; 
SUK: Kolhapur District, Kas, July, M. P. Bachulkar, 5104.

\section{Curcuma scaposa (Nimmo) Skornick. \& M.} Sabu.

\section{Vernacular names: not recorded}

Herbs, root stock tuberous, oblique; root fiber long, slender ending with small root tubers, oval or ellipsoid $1.5-4 \times 0.5-1.5 \mathrm{~cm}$, tubers white inside. Rhizome small, conical, pale brown and white inside, $1.5-4 \times 1-2 \mathrm{~cm}$. Leafy shoot $50-70 \mathrm{~cm}$ long; leaves 4-6, 15-25 × 6-8cm long, oblong lanciolate, acuminate, green, and narrowed, into deeply channeled petiole; petiole shorter than lamina. Inflorescence terminal, $12-15 \mathrm{~cm}$ long; spike 10 $20 \mathrm{~cm}$ long, moderately dense; peduncle long, slender, green; flower white, open at night or early in the morning, 7-9 cm; calyx loosely sheathing corolla tube, 3 toothed, $3-4 \mathrm{~cm}$; corolla tube slender, oblong, 6-8cm long; lateral staminode white; capsule red, obovoid, 1.0-1.2cm long; seeds brown, arillate (Fig. 1j).

*Type: Neotype: (designated by Sornickova et al., 2007: 524): [India, Maharashtra], Western Ghats, Lonavlie, September 1878, G. King s.n. (BM!, isoneo $\mathrm{K}$ !).

Distrib.: Pune, Kolhapur, Ratnagiri

Fls. \& Frts.: July-September

Exsiccata: Lanja, Ratnagiri. ASJ 7614

Specimen examined: B H Mumbai: $K$. scaposa Maharashtra, Lonavala, near Lonavala, 17.09.58, Santapau S.J., 22851, 22852; Khandala, 10.09.1944, H. S., HS 5003; Khandala, 20.09.54, B. Rukminibai, B.R. 253, B.R.254, B.R.268, 197 B.R.; Lonavala, 10.09.54, B. Rukminibai, B.R. 210, B.R. 211; Khandala, 03.09.42, H. S., HS 808(2); Khandala, 2.10.44, H. S., HS 5005, 5006, 5002; Khandala, Sept-Oct., 1918, HS-28213. BAMU: Lonavala, 14.09.1967, Hippalgoankar, 36.
11. Curcuma zedoaria (Christm.) Roscoe

Vernacular names: English: Zedoary, White turmeric Hindi: Jangli haldi, Sanskrit: Shati, Kannada: Kachora, Tamil: Karppurakkilangku, Manipuri: Meitei yaingang, Bengali: Aam aadaa.

Herbs, root tubers many; sessile tubers small, cylindrical, branched $2-2.5 \times 1-1.5 \mathrm{~cm}$ long white inside. Rhizome, pale yellow, bluish at center and white towards periphery, $2.5-5 \times 2-2.7 \mathrm{~cm}$. Leafy shoot 2-3 feet tall or more in length; leaves 4-6, 25$65 \times 10-15 \mathrm{~cm}$ long, oblong lanciolate with acuminate apex, narrowed to the base, purple colored strip on the entire midrib on upper surface of the leaves; petiole shorter than leaf lamina. Inflorescence central, $10-15 \times 3-4 \mathrm{~cm}$; spike 10-15 $\times 2-3.5 \mathrm{~cm}$; flowers yellow shorter than bracts, 2.5 $3.5 \mathrm{~cm}$; comma bract dark pink or shade of others $4.5-5.0 \times 1-2 \mathrm{~cm}$; fertile bract ovate, green, slightly twinged with red 3-4 × 1.5-2 cm; calyx obtusely toothed, whitish; corolla tube funnel shape, $3 \mathrm{~cm}$ long; capsule 3-gonous, smooth, thin, ovoid, dehiscing irregularly; seeds oblong or ellipsoid, with lanciolate, white aril (Fig. 1k).

*Type: Lectotype: (designated by Burtt, 1977: 59): [icon] "Kua" in Rheede, Hort. Malab. 11: t.7.1692.

Distrib: Cultivated Bombay, Chandrapur, Gondia, Raigad and Satara.

Fls. \& Frts.: July- September.

Exsiccata: Amgoan Gondia. ASJ 7624

Specimen examined: BSI: Kollar area, bellow Ponnadi estate, 21.03.1960, R. S. Rao \& N. P. Balkrishnan, 61375; Goa: Molem-Belganm Rd, 17.09.1970, N. Singh \& S. K. Mudliar, 124266; Dudhsagar, 17.09.1970, N. P. Singh \& S. K. Mudliar, 124007.

* Sornickova et al. (2010). 


\section{Key to the species}

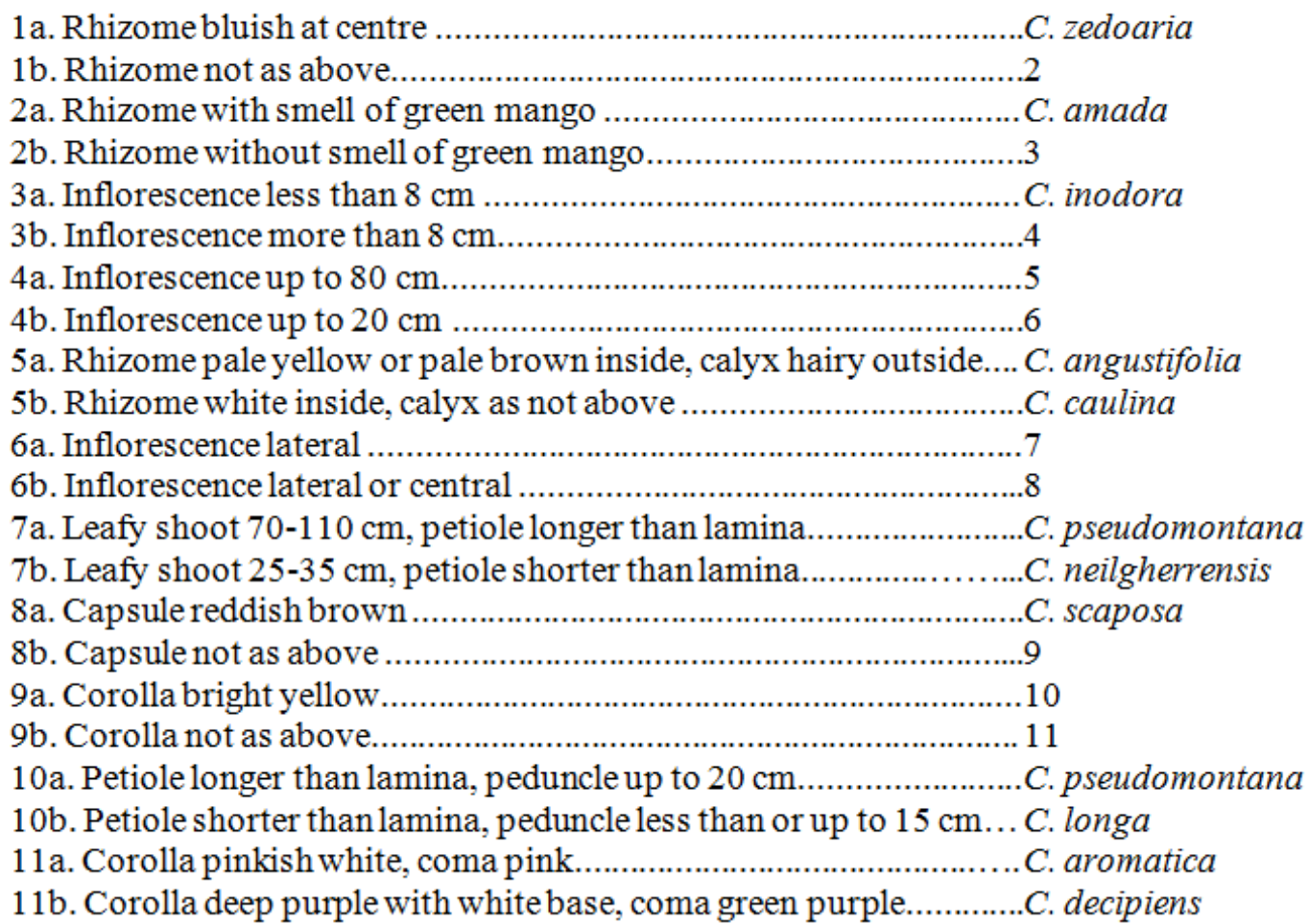

\section{Conclusion}

The study concluded that there are 11 species recorded in Maharashtra state. In case of misidentification in the genera $C$. purpurea Blatt., is reduced here a taxonomic synonym of $C$. inodora Blatt. Hitchenia caulina J. Grahm. is merged in Curcuma and the valid name is $C$. caulina $\mathrm{J}$. Grahm., Kaempferia scaposa (Nimmo) is a synonym of C. scaposa (Nimmo) J. Skornick. \& M. Sabu.

\section{Conflict of interest statement}

Authors declare that they have no conflict of interest.

\section{Acknowledgement}

Authors are thankful to principal of Vivekanand Arts, Sardar Dalipsingh Commerce and Science College, Samarth Nagar, Aurangabad for providing laboratory facilities for the present research work.

\section{References}

Chen, J., Lindstrom, A. J., Xia, N., 2015. Curcuma woodii (Zingiberaceae), a new species from Thailand. Phytotaxa. 227(1), 75-82.

Chen, J., Xia, N., 2013. Curcuma gualinqingensis sp. nov. (Zingiberaceae) from Yunnan China. Nordic J. Bot. 31, 711-716.

Cooke, T., 1958. The Flora of the Presidency of Bombay, Vol. 3. Botanical Survey of India, Calcutta. pp.235-239.

Kress, W. J., Prince, L. M., Williams, K. J., 2002. The phylogeny and new classification of the gingers (Zingiberaceae): Evidence from molecular data. Amer. J. Bot. 89(11), 16821696.

Naik, V. N., 1998. Flora of Marathwada. Vol. II. Amrut Prakashan. pp. 830-832.

Naïve, M. A. K., 2017. Zingiberaceae of Kalatungan Mountain Range, Bukidnon, Philippines. Biosci. Discov. 8(3), 311-319.

Sabu, M., 2006. Zingiberaceae and Costaceae of South India. Indian Association for Angiosperm Taxonomy, Calicut University, India. 34p. 
Sharma, B. D., Karthikeyan, S., Singh, N. P., Lakshminarsimhan, P., 1996. Flora of Maharashtra state Monocotyledons. Botanical Survey of India. pp.72-77.

Skornickova, J., Sabu, M., 2002. The genus Curcuma L. in India: Resume and future prospects. In: Perspective of Plant Biodiversity (Ed.: Das, A.P.). Bishen Singh Mahendrapal
Singh, Deharadun, India. pp.45-51.

Sornickova, L., Otakar, S., Marhold, K., 2010.

Back to types! Towards stability of names in Indian Curcuma L. (Zingiberaceae). Taxon. 59(1), 269-282.

Yadav, S. R., Sardesai, M. M., 2002. Flora of Kolhapur District. Shivaji University Kolhapur (India). pp.476-478.

\section{How to cite this article:}

Jadhao, A. S., Bhuktar, A. S., 2018. Genus Curcuma L. (Zingiberaceae) from Maharashtra State - India. Int. J. Curr. Res. Biosci. Plant Biol. 5(10), 39-48. doi: https://doi.org/10.20546/ijcrbp.2018.510.006 